\title{
Comparison of the Effects of Aerobic Exercise on Pulmonary Function and Levels of Inflammatory Mediators in Men With Type 2 Diabetes
}

\author{
Hossein Saki ${ }^{1}$, Shiva Shahrokhian², Vahid Taeid ${ }^{3}$, Mahmood Amani ${ }^{1}$, Hamid Talebifard ${ }^{4}$, Mojtaba \\ Delaramnasab $^{5^{*}}$
}

${ }^{1}$ MSc in Exercise Physiology, Department of Physical Education and Sports Science, Bu-Ali Sina University, Hamedan, Iran ${ }^{2} \mathrm{MSc}$ in Exercise Physiology, Department of Physical Education and Sports Science, Shahid Chamran University, Ahvaz, Iran ${ }^{3} \mathrm{MSc}$ in Exercise Physiology, Department of Physical Education and Sports Science, Shahid Rajaee Teacher Training University, Tehran, Iran

${ }^{4} \mathrm{MSc}$ in Exercise Physiology, Department of Physical Education and Sports Science, Abadan Branch, Islamic Azad University, Iran

${ }^{5}$ Clinical Research Development Unit, Amir-Al-Momenin Hospital, Zabol University of Medical Sciences, Zabol, Iran

\author{
*Correspondence to \\ Mojtaba Delaramnasab, \\ Tel: +985432232166 \\ Email: delaramnasab@yahoo.com
}

Received May 16, 2017 Accepted June 17, 2017 Published online June 30, 2017

Please cite this article as follows: Saki H, Shahrokhian S, Taeid V, Amani M, Talebifard $H$, Delaramnasab M. Comparison of the effects of aerobic exercise on pulmonary function and levels of inflammatory mediators in men with type 2 diabetes. Int J Basic Sci Med. 2017;2(2):95100. doi:10.15171/ ijbms.2017.18.

\begin{abstract}
Introduction: Insufficient pulmonary function is a complication of type 2 diabetes coordinating with poor blood sugar management and promoting an inflammatory condition. Our objective was to assess the effects of consistent aerobic exercises on pulmonary function and levels of some inflammatory cytokines in males with type 2 diabetes.

Methods: In the present semi-experimental study, 20 men with type 2 diabetes were selected using purposive sampling method. The recruited patients were randomly assigned into one of the aerobic exercise or control groups. The exercises continued for 8 weeks, 3 sessions per week, and each session consisted 45-60 minutes of aerobic exercise with intensity of 50\%-70\% heart rate reserve (HRR). Spirometry and hematologic parameters were both measured at 48 hours prior and 72 hours subsequent to the intervention. Statistical analysis was performed using SPSS v. 22.0 statistical software. Independent and paired sample $t$ test were used for inferential analysis with $P \leq 0.05$ regarded as statistically significant.

Results: A significant reduction was observed in serum levels of fasting blood sugar (FBS), glycosylated hemoglobin (HbA1c), C-reactive protein (CRP), and interleukin 6 (IL-6) in aerobic exercise group $(P<0.05)$. On the other hand, forced vital capacity $(\mathrm{FVC})$, and forced expiratory volume (FEV1) levels showed a significant elevation in the experimental group relative to the control.

Conclusion: Considering our findings, it seems that aerobic exercise can improve pulmonary function in type 2 diabetes patients. This may be in some levels mediated by stabilizing blood glucose levels and subsiding systemic inflammatory condition in these patients.

Keywords: Type 2 diabetes, Aerobic exercise, Inflammation, Pulmonary function
\end{abstract}

415 million people affected with diabetes across the globe from which $90 \%$ are type 2 diabetes patients. Development of type 2 diabetes has been correlated with obesity, unhealthy lifestyle, and increasing age. ${ }^{4}$ Poor management of blood glucose results in endothelial complications and reduced pulmonary functional indices. In this regard, limited air flow has been noted as a risk factor, predicting death. ${ }^{5}$ Studies have shown that general disorders in pulmonary distal regions including alveolar membrane

Copyright (C) 2017 The Author(s); Published by Zabol University of Medical Sciences. This is an open-access article distributed under the terms of the Creative Commons Attribution License (http://creativecommons.org/licenses/by/4.0), which permits unrestricted use, distribution, and reproduction in any medium, provided the original work is properly cited. 
and distal respiratory tract may be related to pulmonary hyperemia and insufficiency of high respiratory output. These in turn have been associated with obesity. ${ }^{6}$ Inactivity and obesity are 2 contributors to the insufficiency of respiratory system. There have been notions that high body fat content and overweight negatively influence pulmonary function. These detrimental effects may be mediated by alternations in respiratory mechanisms, reduction in muscular strength and stamina, lowering the rate of pulmonary gas exchange and limiting exercise tolerance. $^{7,8}$ Mechanisms leading to the pulmonary damage in diabetes patients are not yet well-understood. However, evidence from pathological studies suggests that the magnificent histological changes especially altered wall thicknesses of alveoli, capillaries, and also pulmonary arterioles are responsible for pulmonary dysfunction in diabetes patients. ${ }^{9}$ Hypercalcemia and oxidative stress can promote abnormal function of pulmonary muscles in diabetes which is a risk factor of pulmonary distress conditions. ${ }^{10}$ Diabetes has been associated with the dysfunction of diaphragm muscle and significantly reduced function of respiratory muscles.

The possible mechanism for the reduced strength of diaphragm (the main respiratory muscle) has been proposed to be the destruction of axon of phrenic nerve in diabetes patients. ${ }^{11-13}$ Hyperglycemia and glycosylation of chest wall have been suggested as the main causes of pulmonary dysfunctions in diabetes patients. Nevertheless, some studies have reported that higher rate of respiratory infections, inflammation, and diabetes-induced oxidative stress are involved in the reduced function of respiratory muscles and pulmonary insufficiency. ${ }^{14}$

Another parameter leading to the pulmonary dysfunction is systemic inflammation. ${ }^{15}$ Kritikou et al showed in their research that inflammatory mediators including C-reactive protein (CRP), interleukin 6 (IL-6), and insulin resistance may be involved in respiratory abnormalities in middle-aged men and women. ${ }^{16}$ Patients with type 2 diabetes have been reported to suffer from a low-grade systemic inflammation represented by increased levels of acute phase proteins, and inflammatory cytokines such as CRP and IL- $6 .{ }^{17,18}$ There are arguments regarding the role of IL-6 in insulin resistance. In humans, IL-6 level may be correlated with insulin resistance. ${ }^{19}$ One of the major functions of IL-6 is the induction of CRP production. ${ }^{20}$ CRP is a biomarker of systemic inflammation released in response to the accumulation of inflammatory cytokines. ${ }^{21}$ Higher levels of CRP have been associated with higher blood pressure, increased body mass index (BMI), metabolic syndrome, diabetes, dyslipidemia, as well as chronic infectious and inflammatory conditions. ${ }^{22}$ Regular exercise represents anti-inflammatory effects and suppresses low-grade systemic inflammation. ${ }^{23}$ Studies have shown that alternation in lifestyle such as lowering calorie intake and increasing physical activity can alleviate inflammatory markers. ${ }^{24}$ Kasapis and Thompson in their review study regarding the effects of exercise and physical activity on inflammation concluded that regular physical activity could significantly lower inflammatory state and CRP level. ${ }^{25}$ Subsequently, systemic and pulmonary inflammation could be placated. ${ }^{24}$

In comparison to the large body of studies addressing cardiovascular conditions, nephropathy, retinopathy, and neuropathy in diabetes, there are few numbers of studies on pulmonary complications. ${ }^{26}$ This necessitates studies to address this issue in diabetes patients. Regarding the reduced pulmonary capacity in type 2 diabetes patients, ${ }^{27}$ and positive effects of physical activity on improving physical fitness and especially aerobic function of lungs, it seems that physical exercise, especially persistent medium-intensity aerobic exercise, could be one of the therapeutic and precautionary modalities for reducing inflammation and improving pulmonary function in diabetic patients. In line with this view, previous studies have indicated an influential role for aerobic exercise in improving the pulmonary function in type 2 diabetes patients. ${ }^{26-28}$ Bearing in the mind the role of inflammatory process in pulmonary dysfunction, and inconsistencies of the results about the role of aerobic exercise in respiratory function in a chronic disease like diabetes, we aimed to evaluate the effects of persistent aerobic exercise on inflammatory markers of IL-6 and CRP, and pulmonary function in patients affected by type 2 diabetes.

\section{Methods}

In the present one-sided blind semi-experimental study, 40 men with type 2 diabetes, aged 35-50 were recruited. The patients had fasting blood sugar (FBS) levels of 125$200 \mathrm{mg} / \mathrm{dL}$ and BMI of $25-30 \mathrm{~kg} / \mathrm{m}^{2}$. They were selected based on a targeted sampling method and randomly assigned into 2 groups of aerobic exercise and control.

\section{Exercise Protocol}

The aerobic exercise was performed within 8 weeks, 3 sessions per week, and each session lasted 45-60 minutes. The exercises comprised running with intensity of $50 \%-70 \%$ heart rate reserve (HRR) in a gym. HRR was calculated according to the Karvonen formula, and carotid pulse rate was used for screening the heart rate during the exercise. ${ }^{29}$ The exercise protocols used in the current study were author-based, and prepared based on the effectiveness of aerobic exercises on pulmonary functional volumes in patients with type 2 diabetes. This protocol was a modified protocol previously used by Oluwaseyi et al in type 2 diabetes patients. ${ }^{5}$ In this program, warm-up initiated at first as gentle aerobic exercise including walking and jogging, as well as stasisdynamic warm-up exercises. Afterwards, the main exercise protocol was performed including walking and continuous running for 30-45 minutes. Intensity of the exercise was 50\%-60\% HRR in first weeks, and 60\%-70\% HRR in the weeks of 5-8. Recovery exercises including gentle warms-up and aerobic exercises were performed for 10 minutes following the main program (Table 1). 
Table 1. Detailed Aerobic Exercise Protocol for Type 2 Diabetes Patients

\begin{tabular}{llll}
\hline Time Course of the Study & & Time (min) & Extent and Type of Exercises \\
\hline \multirow{3}{*}{ First 4 weeks } & Warm-up exercise (walking and jogging) & 10 & $50 \%-60 \%$ HRR \\
& Aerobic exercise (continuous running) & $30-45$ & 5 \\
& Recovery exercise (gentle warms-up) & 10 \\
Second 4 weeks & Warm-up exercise (walking and jogging) & $30-45$ \\
& Aerobic exercise (continuous running) & $50-70 \%$ HRR \\
\hline
\end{tabular}

Abbreviation: HRR, heart rate reserve.

Biochemical Measurements

Laboratory measurements of biochemical indices were performed in 2 occasions; 48 hours before the intervention and 72 hours after the last session of the intervention. The measurements were made in early morning fasting state (8-9 AM). Blood samples ( $8 \mathrm{~mL}$ ) were obtained from the radial veins of the right hand. FBS level was measured using Pars Azmoun Eliza kit (Iran) which rendered $1 \mathrm{mg} / \mathrm{dL}$ sensitivity. CRP was measured by immunoturbidity method using auto analyzer BS-380 with detection limit of $1 \mathrm{pg} / \mathrm{dL}$. IL-6 level was determined using Eliza kit manufactured by Biovendor Company (Germany) with the sensitivity of $0.92 \mathrm{pg} / \mathrm{mL}$. Pulmonary functional parameters were assessed using spirometry instrument (IF-9 mode, Germany) in a sitting position and in triplicate. The best functions were documented as the output. Measurement of the maximum consumed oxygen following spirometry test was made by Storer Maximal Bicycle test. ${ }^{30}$

\section{Statistical Analyses}

Statistical analysis was performed by student $t$ test in SPSS v. 22.0 statistical software. We used independent sample $t$ test and paired sample $t$ test for the evaluation of means differences for within and between analyses. Statistical significance level was $P=0.05$.

\section{Results}

Table 2 shows the demographic variables (age, weight, $\mathrm{BMI}, \mathrm{VO}_{2 \max }$, and history of the disease) in the studied groups. Independent sample student $t$ test showed no significant difference between the groups at the baseline $(P>0.05)$.

Results of paired sample $t$ test (Table 3) revealed a meaningful reduction in FBS level $(20.75 \mathrm{mg} / \mathrm{dL}, 13.9 \%$, $P<0.001)$, HbA1c $(0.69 \%, 9.7 \%, P<0.001)$, IL-6 (0.48 $\mathrm{mg} / \mathrm{dL}, 9.8 \%, P=0.001)$, and CRP (0.6 pg/dL, 10.3\%, $P=0.003)$, a significant elevation in forced vital capacity (FVC) $(0.16 \mathrm{~L}, 4.6 \%, P=0.006)$, and forced expiratory volume (FEV1) $(0.13 \mathrm{~L}, 4.2 \%, P=0.011)$ in the aerobic exercise group. A significant increase was also observed for IL-6 $(0.12 \mathrm{mg} / \mathrm{dL}, 2.2 \%, P<0.028)$, and CRP $(0.13$ $\mathrm{pg} / \mathrm{dL}, 2.2 \%, P=0.001)$ in the control group. In between group analysis, significant difference was observed for differences of FBS $(P=0.001)$, HbA1c $(P=0.003)$, IL-6 $(P<0.001)$, CRP $(P=0.004)$, FVC $(P=0.001)$, and FEV1 $(P<0.01)$.

\section{Discussion}

In the present study, a significant reduction in the levels of CRP, IL-6, and HbA1c was observed following the 8 weeks of aerobic exercise compared to the control group. This reduction in the inflammatory markers following the exercise intervention was in line with some previous reports. ${ }^{18,25,31,32}$ Exercise represents a natural method for regulating metabolism, blood sugar, and systemic inflammation. ${ }^{23}$ Increased serum levels of IL- 6 has been correlated with the progression of type 2 diabetes and insulin resistance. ${ }^{33}$ Previous studies have reported that exercise can lead to the release of multiple cytokines including IL-6 into blood stream from active muscles. This in turn can mediate metabolic alternations in the body. ${ }^{34}$ In contrast to the IL- 6 produced by T-lymphocytes and macrophages, the cytokine produced by muscle renders anti-inflammatory properties. ${ }^{35}$ On the other hand, it has been noted that increased epinephrine as a result of exercise can downregulate tumor-necrosis factor (TNF- $\alpha$ ) response leading to subsided inflammatory state. $^{36}$ Regarding the relationship between insulin resistance and inflammation, ${ }^{16,19}$ it is comprehensible that the exercise has resulted in the reduction of inflammatory markers of IL- 6 and CRP mediated by exercises through

Table 2. Demographic Characteristics and Pre-test Evaluated Variables

\begin{tabular}{lccrc}
\hline Variables & $\begin{array}{c}\text { Aerobic exercise } \\
\text { Mean } \pm \text { SD }\end{array}$ & $\begin{array}{c}\text { Control } \\
\text { Mean } \pm \text { SD }\end{array}$ & $\boldsymbol{t}$ \\
\hline Age $(\mathrm{y})$ & $4.06 \pm 44.25$ & $4.86 \pm 43.50$ & 1.557 & 0.142 \\
Weight $(\mathrm{kg})$ & $6.05 \pm 72.74$ & $10.62 \pm 76.50$ & -0.528 & -2.027 \\
$\mathrm{BMI}\left(\mathrm{kg} / \mathrm{m}^{2}\right)$ & $1.40 \pm 26.73$ & $1.57 \pm 27.50$ & -1.224 & 0.606 \\
$\mathrm{VO}_{2 \mathrm{max}}(\mathrm{mL} / \mathrm{kg} /$ Min) & $3.12 \pm 31.08$ & $1.30 \pm 32.54$ & -0.333 & 0.241 \\
Time from diagnosis $(\mathrm{y})$ & $1.49 \pm 6.25$ & $1.51 \pm 6.50$ & 0.744 \\
\hline
\end{tabular}

Abbreviations: BMI, body mass index. 
Table 3. Values of Measured Variables Between and Within Groups

\begin{tabular}{|c|c|c|c|c|c|c|c|}
\hline \multirow{2}{*}{ Variables } & & \multicolumn{4}{|c|}{ Paired Sample $t$ test } & \multicolumn{2}{|c|}{ Independent Sample $t$ test } \\
\hline & & $\begin{array}{c}\text { Pre-test } \\
(\text { Mean } \pm \text { SD })\end{array}$ & $\begin{array}{c}\text { Post-test } \\
(\text { Mean } \pm \text { SD })\end{array}$ & $t$ & $P$ & $\begin{array}{c}\Delta(\text { Mean } \\
\text { Difference } \pm \text { SD) }\end{array}$ & $P$ \\
\hline \multirow{2}{*}{$\mathrm{FBS}(\mathrm{mg} / \mathrm{dL})$} & Training & $149.13 \pm 17.92$ & $128.38 \pm 15.46$ & 6.588 & $0.0001^{b}$ & $-20.75 \pm 8.91$ & \multirow{2}{*}{$0.001^{b}$} \\
\hline & Control & $153.13 \pm 17.92$ & $152.25 \pm 17.92$ & 0.262 & 0.801 & $-0.88 \pm 9.45$ & \\
\hline \multirow{2}{*}{ HbA1c(\%) } & Training & $7.08 \pm 0.62$ & $6.39 \pm 0.53$ & 6.945 & $0.0001^{b}$ & $-0.69 \pm 0.28$ & \multirow{2}{*}{$0.003^{a}$} \\
\hline & Control & $7.31 \pm 0.41$ & $7.18 \pm 0.63$ & 1.036 & 0.335 & $-0.13 \pm 0.34$ & \\
\hline \multirow{2}{*}{$\mathrm{FVC}(\mathrm{L})$} & Training & $3.46 \pm 0.53$ & $3.62 \pm 0.48$ & -3.870 & $0.006^{\mathrm{a}}$ & $+0.16 \pm 0.12$ & \multirow{2}{*}{$0.004^{a}$} \\
\hline & Control & $3.56 \pm 0.37$ & $3.51 \pm 0.36$ & 1.080 & 0.316 & $-0.05 \pm 0.13$ & \\
\hline \multirow{2}{*}{ FEV1 (L) } & Training & $3.08 \pm 0.45$ & $3.21 \pm 0.45$ & -3.416 & $0.011^{\mathrm{a}}$ & $+0.13 \pm 0.10$ & \multirow{2}{*}{$0.011^{\mathrm{a}}$} \\
\hline & Control & $3.06 \pm 0.42$ & $3.05 \pm 0.39$ & 0.424 & 0.685 & $-0.01 \pm 0.08$ & \\
\hline \multirow{2}{*}{ IL-6 (mg/dL) } & Training & $4.86 \pm 0.27$ & $4.38 \pm 0.43$ & 5.760 & $0.001^{\mathrm{a}}$ & $-0.48 \pm 0.24$ & \multirow{2}{*}{$0.0001^{b}$} \\
\hline & Control & $5.33 \pm 0.42$ & $5.45 \pm 0.39$ & -2.257 & $0.028^{\mathrm{a}}$ & $+0.13 \pm 0.12$ & \\
\hline \multirow{2}{*}{ CRP (pg/dL) } & Training & $5.81 \pm 0.61$ & $5.21 \pm 0.47$ & 4.583 & $0.003^{\mathrm{a}}$ & $-0.60 \pm 0.37$ & \multirow{2}{*}{$0.001^{b}$} \\
\hline & Control & $5.78 \pm 0.52$ & $5.91 \pm 0.51$ & -3.274 & $0.014^{\mathrm{a}}$ & $+0.14 \pm 0.11$ & \\
\hline
\end{tabular}

Abbreviations: $\triangle$, Pretest - posttest; FBS, fasting blood sugar; HbA1C, hemoglobin A1C; FVC, forced vital capacity; FEV1, forced expiratory volume in the first second; IL-6, Interleukin- 6; CRP, C-reactive protein.

${ }^{\text {a }} P<0.05$; ${ }^{\text {b }} P \leq 0.001$.

the regulation of blood sugar (evidenced by lower FBS and $\mathrm{HbA1c}$ ).

In the present study, a significant elevation was recorded for FEV1 and FVC following the 8 weeks of aerobic exercises. In line with our results, significant increases in the FEV1 and FVC serum levels of type 2 diabetic patients were also reported by Ghalavand et al (following the 8 weeks of aerobic exercises), ${ }^{26}$ Afshonpour et al (following the 8 weeks of combined aerobic-resistance exercises), ${ }^{28}$ and Oluwaseyi et al (following the 12 weeks of combined aerobic-resistance exercises). ${ }^{5}$ In contrast to our results, however, Ghalavand et al observed no significant alternation in FEV1 and FVC levels in type 2 diabetic men after 8 weeks of circuit resistance training. This disagreement may be explained by different exercise programs in our study. It seems that incorporating aerobic exercise in therapeutic protocols can have beneficial effects on augmenting pulmonary function in type 2 diabetes patients.

FVC is a pulmonary functional volume dependent on age, physical activity index, body composition, and health states of individuals. ${ }^{37,38}$ FVC rate which reflects the strength of respiratory muscles is determined by the pulmonary elasticity and resistance of respiratory tracts. In addition, FVC is dependent on the respiratory muscles' strength and the acceptance level of chest., ${ }^{5,926}$ FEV1 is another important indicator of pulmonary function. This factor is reduced in response to multiple conditions such as low-physical fitness and diabetes. FEV1 reduction may indicate diminished total lung capacity, respiratory airway obstruction, loss of pulmonary reversibility capacity, and less common insufficient respiratory muscular development. ${ }^{9}$ With promoted strength and resistance of respiratory muscles following the exercise, pulmonary functional volumes such as FVC and FEV1 raise in diabetic subjects. Such elevation in the present study can be related to the role of aerobic exercise in preserving the blood sugar. ${ }^{26,28}$ It seems that controlling the blood sugar in diabetes patients can lead to the improvement of pulmonary function in these patients. Regarding the role of inflammation in pulmonary dysfunction, ${ }^{15}$ increased FVC and FEV1 levels in the present study may be explainable in part by reduced systemic inflammation markers (IL-6 and CRP). Other factors influencing FVC and FEV1 spirometry indices are augmented strength and endurance of respiratory muscles including diaphragm, inter-tibia muscles, and abdominal muscles, as well as reduced resistance of respiratory air-ways, and improved elasticity and reversibility of lung tissue. ${ }^{28}$

Despite numerous studies on diabetes complications and interventional based management of these compilations, studies on pulmonary complications are limited in this area. Small sample size and short-intervention period are among the limitations ofthese studies. In general, our findings highlighted that 8 weeks of aerobic exercise can reduce the levels of inflammatory markers of IL-6 and CRP, increase the spirometry parameters of FVC and FEV1, and improve pulmonary function. All of these effects seem to be mediated by maintenance of blood sugar level.

\section{Conclusion}

Considering our findings, it seems that aerobic exercise can improve pulmonary function in type 2 diabetes patients. This may be in some level mediated by stabilizing blood glucose levels and subsiding systemic inflammatory condition in these patients. It is recommended to incorporate regular aerobic exercise protocols in therapeutic regimen in type 2 diabetes to help patients in management of their disease.

\section{Ethical Approval}

This research has been ethically approved by Hamedan University under the code of 8823 . 


\section{Competing Interests}

Authors declare that they have no potential conflict of interests.

\section{References}

1. Bhurosy T, Jeewon R. Overweight and obesity epidemic in developing countries: a problem with diet, physical activity, or socioeconomic status? ScientificWorldJournal. 2014;2014:964236. doi.org/10.1155/2014/964236

2. Nazem F, Izadi M, Keshavarz B, Jalili M. Impact of aerobic exercise and detraining on pulmonary function indexes in obese middle-aged patients with chronic asthma. Arak Medical University Journal. 2013;15(68):85-93.

3. Bakhshi-Ganje M, Shekhha MH, Farashahi E, Mohammadzade M. Association of rs16953002 FTO Gene polymorphism with type 2 diabetes and obesity. J Fasa Univ Med Sci. 2016;6(1):27-34.

4. Chatterjee S, Khunti K, Davies MJ. Type 2 diabetes. Lancet. 2017 Feb 10

5. Oluwaseyi O, Sunday A, Abraham O, Olajide O. Effect of progressive aerobic and resistance exercises on the pulmonary functions of individuals with type 2 diabetes in Nigeria. Int J Endocrinol Metab. 2012;2012:411-7. doi: 10.5812/ijem. 3333

6. Oppenheimer BW, Berger KI, Ali S, et al. Pulmonary vascular congestion: a mechanism for distal lung unit Dysfunction in Obesity. PloS One. 2016;11(4):e0152769. doi:10.1371/journal.pone.0152769.

7. Malini M, Baljoshi V, Kammar K. A comparitive study of impact of obesity on static lung volumes and capacities in young adult women. Int J Physiol. 2017;5(1):105-109.

8. Costa D, Barbalho MC, Miguel GP, Forti EM, Azevedo JL. The impact of obesity on pulmonary function in adult women. Clinics. 2008;63(6):719-724. doi:10.1590/S180759322008000600002.

9. Davis WA, Knuiman M, Kendall P, Grange V, Davis TM. Glycemic exposure is associated with reduced pulmonary function in type 2 diabetes. Diabetes Care. 2004;27(3):752757. doi:10.2337/diacare.27.3.752

10. Acharya PR, D'Souza M, Anand R, Kotian SM. Pulmonary function in type 2 diabetes mellitus: correlation with body mass index and glycemic control. International Journal of Scientific Study. 2016;3(11):18-23.

11. Andersen H, Nielsen S, Mogensen CE, Jakobsen J. Muscle strength in type 2 diabetes. Diabetes. 2004;53(6):15431548. doi:10.2337/diabetes.53.6.1543.

12. Kabitz H-J, Sonntag F, Walker D, et al. Diabetic polyneuropathy is associated with respiratory muscle impairment in type 2 diabetes. Diabetologia. 2008;51(1):191-197. doi:10.1007/s00125-007-0856-0.

13. Corrêa AP, Ribeiro JP, Balzan FM, Mundstock L, Ferlin EL, Moraes RS. Inspiratory muscle training in type 2 diabetes with inspiratory muscle weakness. Med Sci Sports Exerc. 2011;43(7):1135-1141. doi:10.1249/ MSS.0b013e31820a7c12.

14. Malek F, Malek M, Tosi J, Soltabi S, Hashemi H. Comparison of pulmonary function in diabetic patients with and without retinopathy compared with control group. J Endocrinol Metab. 2010;11(2):143-150.

15. Baines KJ, Backer V, Gibson PG, Powel H, Porsbjerg CM. Impaired lung function is associated with systemic inflammation and macrophage activation. Eur Respir J.
2015;45(2):557-559. doi:10.1183/09031936.00187514.

16. Kritikou I, Basta M, Vgontzas AN, et al. Sleep apnoea, sleepiness, inflammation and insulin resistance in middleaged males and females. Eur Respir J. 2014;43(1):145-155. doi:10.1183/09031936.00126712.

17. Zeng C, Shi X, Zhang B, et al. The imbalance of Th17/Th1/ Tregs in patients with type 2 diabetes: relationship with metabolic factors and complications. J Mol Med (Berl). 2012;90(2):175-186. doi:10.1007/s00109-011-0816-5.

18. Zamanpour L, Banitalebi E, Amirhosseini S. The effect of sprint training and combaned aerobic and strenght training on som inflammatory markers and insulin resistance in women with type 2 diabetes mellitus (T2DM). Iranian Journal of Diabetes and Metabolism. 2016;15(5):300-311.

19. Lukic L, Lalic NM, Rajkovic N, et al. Hypertension in obese type 2 diabetes patients is associated with increases in insulin resistance and IL- 6 cytokine levels: potential targets for an efficient preventive intervention. Int $J$ Environ Res Public Health. 2014;11(4):3586-3598. doi:10.3390/ ijerph110403586

20. Darabi S, Hasanvand A, Nourollahi A. Assessment of the effects of anti-inflammatory of garlic; nettle leaves and olives extracts in STZ-induced diabetic rat. Complementary Medicine Journal of Faculty of Nursing \& Midwifery. 2016;6(1):1451-1460.

21. Swift DL, Johannsen NM, Earnest CP, Blair SN, Church TS. The effect of exercise training modality on C-reactive Protein in Type-2 Diabetes. Medicine and science in sports and exercise. 2012;44(6):1028. doi:10.1249/ MSS.0b013e31824526cc

22. Mugabo Y, Li L, Renier G. The connection between C-reactive protein (CRP) and diabetic vasculopathy. Focus on preclinical findings. Curr Diabetes Rev. 2010;6(1):2734. doi:10.2174/157339910790442628.

23. Petersen AMW, Pedersen BK. The anti-inflammatory effect of exercise. J Appl Physiol. 2005;98(4):1154-1162. doi:10.1152/japplphysiol.00164.2004.

24. Scott H, Gibson P, Garg M, et al. Dietary restriction and exercise improve airway inflammation and clinical outcomes in overweight and obese asthma: a randomized trial. Clin Exp Allergy. 2013;43(1):36-49. doi:10.1111/ cea. 12004 .

25. Kasapis C, Thompson PD. The effects of physical activity on serum C-reactive protein and inflammatory markers: a systematic review. J Am Coll Cardiol. 2005;45(10):15631569. doi:10.1016/j.jacc.2004.12.077.

26. Ghalavand A, Shakeryan S, Nikbakht M, Mehdipour A, Monazamnezhad A, Delaramnasab M. Effects of aerobic training on cardiorespiratory factors in men with type 2 Diabetes. J Diabetes Nurs. 2014;2(2):8-17.

27. Aparna A. Pulmonary function tests in type 2 diabetics and non-diabetic people-a comparative study. J Clin Diagn Res. 2013;7:1606-1608. doi:10.7860/JCDR/2013/6182.3237.

28. Afshonpour M, Ghalavand A, Rezaee R, Habibi A. The effect of exercise training on pulmonary function in type 2 diabetic men. Alborz University Medical Journal. 2015;4(4):255-265.

29. Ghalavand A, Delaramnasab M, Sayari A, Heydari, Rostami A. The effect of resistance training on cardiaometabolic factors in men with type 2 diabetes. Quarterly Journal of Caspian Health and Aging. 2016;1(2):15-21.

30. Abolfathi F, Ranjbar R, Shakerian S, Yazdanpanah L. The effect of eight weeks aerobic interval training on adiponectin 
serum levels, lipid profile and HS-CRP in women with type II diabetes. Iranian Journal of Endocrinology and Metabolism. 2015;17(4):316-324.

31. Hayashino Y, Jackson JL, Hirata $\mathrm{T}$, et al. Effects of exercise on C-reactive protein, inflammatory cytokine and adipokine in patients with type 2 diabetes: a metaanalysis of randomized controlled trials. Metabolism. 2014;63(3):431-440.

32. Kim KB. Effect of different training mode on Interleukin-6 (IL-6) and C-reactive protein (CRP) in type 2 diabetes mellitus (T2DM) patients. J Exerc Nutrition Biochem. 2014;18(4):371. doi:10.5717/jenb.2014.18.4.371

33. Yousefipoor P, Tadibi V, Behpoor N, Parnow A, Delbari E, Rashidi S. The effect of 8-week aerobic and concurrent (aerobic- resistance) exercise training on serum IL-6 levels and insulin resistance in type 2 diabetic patients. Journal of Shahid Sadoughi University of Medical Sciences. 2013;21(5):619-631.

34. Jalili M, Heydarianpour A. The effect of L-carnitine and
10 weeks of aerobic exercise on serum levels of CRP in streptozotocin-induced diabetic rats. Iranian Journal of Endocrinology and Metabolism. 2016;17(6):435-439.

35. Beavers KM, Brinkley TE, Nicklas BJ. Effect of exercise training on chronic inflammation. Clinica Chimica Acta. 2010;411(11):785-793. doi:10.1016/j.cca.2010.02.069.

36. Golbidi S, Badran M, Laher I. Antioxidant and antiinflammatory effects of exercise in diabetic patients. Exp Diabetes Res. 2011;2012:941868. doi:10.1155/2012/941868.

37. Ghalavand A, Shakeriyan S, Rezaee R, Hojat S, Sarshin A. The effect of resistance training on cardio respiratory factors in men with type 2 diabetes. Alborz University Medical Journal. 2015;4(1):59-67.

38. Womack CJ, Harris DL, Katzel LI, Hagberg JM, Bleecker ER, Goldberg AP. Weight loss, not aerobic exercise, improves pulmonary function in older obese men. J Gerontol A Biol Sci Med Sci. 2000;55(8):M453-M457. doi:10.1093/ gerona/55.8.M453 